\title{
Is sub-commissural annuloplasty a safe adjunct to sutureless Perceval-S aortic valve implantation?
}

\author{
Aurélien Roumy ${ }^{1}$, Mario Verdugo ${ }^{1}$, ziyad Gunga ${ }^{1}$, Pierre Monney ${ }^{1}$, Valentina Rancati ${ }^{1}$, \\ and Matthias Kirsch ${ }^{1}$ \\ ${ }^{1} \mathrm{CHUV}$
}

June 4, 2020

\begin{abstract}
Background: Sutureless bioprothesis aortic valves simplify surgery for aortic valve replacement (AVR) but some anatomical features of the recipients aortic annulus might preclude anchoring and lead to paravalvular leak. Sub-commissural annuloplasty (SCAP) has been sporadically proposed to secure implantation under these circumstances. This study evaluated whether SCAP affects early postoperative outcomes after sutureless Perceval-S implantation. Methods: We included all patients who underwent AVR (isolated or combined with coronary bypass) with the Perceval-S valve from March 2016 to August 2019. SCAP was performed each time the surgeon deemed it useful to improve anchoring, including after primary implantation failure. Results: One hundred and three patients were included. Mean age was 73.9 \pm 7.2 years and 36(35\%) were women. SCAP was performed in 34(33\%) patients, significantly more frequently in patients with large aortic annulus or bicuspid aortic valve. Perceval-S implantation was successful in 100(97\%) patients and SCAP allowed successful redeployment of the same Perceval S valve in 4(28\%) of first implantation failures. Thirty-day mortality was $2 \%(n=2)$, of which one was related to the procedure. There was no significant difference in the incidence of postoperative conduction disorders between patients with and without SCAP (respectively, 3[9\%] vs $7[10 \%], \mathrm{p}=1.0$ ). More than trivial paravalvular leak was noted in $1(1 \%)$ patient without SCAP. Postoperative peak and mean pressure gradients were lower in patients with than in those without SCAP (respectively, 19.0 \pm 7.9 $\mathrm{mmHg}$ vs $23.1 \pm 7.0 \mathrm{mmHg}, \mathrm{p}=0.001$; and $10.4 \pm 4.1$ versus $12.6 \pm 3.7 \mathrm{mmHg}, \mathrm{p}=0.001)$. Conclusions: $\mathrm{SCAP}$ is a safe, simple and reproducible technique that might facilitate Perceval-S aortic valve implantation.
\end{abstract}

\section{Introduction}

To date, aortic valve replacement (AVR) is the only curative therapy to treat aortic valve stenosis. Despite the emergence of the transcatheter aortic valve implantation (TAVI) procedure, surgical approach remains the gold standard, especially because it allows for the removal of the diseased valve and decalcification of the annulus, optimizing the annulus size and limiting paravalvular leak occurrence. Over the past decade, sutureless and rapid deployment aortic valves have emerged, offering an alternative to conventional AVR.

The Perceval-S aortic valve (LivaNova, London, UK) is a bioprosthesis based on bovine pericardial leaflets mounted into a flexible, self-expandable nitinol stent. This is the only sutureless valve available on the market and presents several advantages. First, its implantation is rapid, simple and reproducible, which reduces aortic cross-clamping time ${ }^{1-4}$. Moreover, its collapsible design favors minimally invasive surgical approaches ${ }^{5}$ and facilitates implantation in challenging situations such as redo operations or calcified aortic root $^{3,6,7}$. It also provides lower transvalvular gradients than conventional stented bioprothesis ${ }^{1,4,8}$. Recent studies have shown that Perceval-S' rate of adverse events (notably renal insufficiency and blood transfusion) are similar or lower to that of conventional bioprothesis ${ }^{1-3}$, while mortality and paravalvular leak rates are lower than in $\mathrm{TAVI}^{3,9-12}$. 
Even if the Perceval-S seems to be attractive and has a broad spectrum of use and advantages, its design based on two anchoring sites (the first at the annulus level and the second at the sino-tubular junction [STJ] level) has some pitfalls. Consequently, Perceval-S is contraindicated in case of a ratio between the STJ and the aortic annulus greater than 1.3, aneurysmal dilation or dissection of the ascending aortic wall ${ }^{13}$. The shape of the annulus is also crucial. In the bicuspid aortic valve (BAV), for example, an ovoid or scalloped annulus (with unequal sub-commissural height) may preclude the valve anchoring and lead to paravalvular leak or valve migration. In this situation, sub-commissural annuloplasty (SCAP) has been sporadically performed in order to circularize the annulus before implanting a Perceval-S ${ }^{14,15}$. In our surgical experience, we regularly challenge non-circular annulus and the aim of this study is to evaluate if SCAP can safely address this issue.

\section{Patients and Methods}

This is a monocentric retrospective study approved by the ethical committee (CER-VD) under the number 2017-00340. From March 2016 to August 2019, all of the patients who benefited from Perceval-S valve implantation in our institution were prospectively considered. The choice to use a Perceval-S valve was left to the operating surgeon, depending on personal preference and the anatomical characteristics cited above. Exclusion criteria were patients $<65$ years old, anatomical features outside the manufacturer's recommendations ${ }^{13}$, Sievers type $0 \mathrm{BAV}$, emergencies and combined surgery other than AVR plus coronary artery bypass graft (CABG). Redo operation was not an exclusion criterion.

\section{Perceval S Implantation Technique}

Procedures were mainly performed through median sternotomy or, alternatively, via an upper J ministernotomy. After starting cardiopulmonary bypass $(\mathrm{CPB})$, a transverse aortotomy was made around $1.5 \mathrm{~cm}$ above the STJ. The aortic valve cups were excised and the annulus conscientiously decalcified to be sufficiently flexible while avoiding annulus lesion. The size of the Perceval-S valve was chosen according to the dedicated sizer and the manufacturer's recommendations ${ }^{13}$. The Perceval-S valve was collapsed into the delivery system and positioned using three guiding sutures placed $1 \mathrm{~mm}$ below the nadir of the aortic valve cusps. These guiding sutures were retrieved after valve expansion. The correct position of the valve inflow ring on the aortic annulus was visually checked. Dilatation with the dedicated balloon was then performed for 30 seconds (either at 2 or $4 \mathrm{~atm}$ ) at $37^{\circ} \mathrm{C}$. The aortotomy was closed using a double Blalock running suture, and after deairing the heart, the aortic clamp was removed and the CPB weaned. Intraoperative transesophagal echocardiography (TEE) confirmed the good position and functioning of the valve, as well as the absence of paravalvular leak prior to CPB removal.

Sub-Commissural Annuloplasty (SCAP)

SCAP consisted of one or two sub-commissural triangle plications (Figure 1), performed by X-stitches using braided Ethibon 2/0: the first one was usually placed under the commissure between the left coronary (LC) and non-coronary (NC) cusps, eventually completed by additional sutures under the commissure between the NC and the right coronary (RC) cusps or the LC and RC cusps. Thus, SCAP permits to reduce slightly the size of the aortic annulus, to circularize an elliptic annulus or to reshape the annulus in the horizontal plane by reducing discrepancies between sub-commissural triangle heights.

\section{Primary Implantation Failure}

Primary implantation failure was defined as the presence of an obvious malposition or instability after valve release before closing the aortotomy, or as a more-than-trivial paravalvular leak after the aorta was unclamped.

In case of implantation failure, the surgeon could (i) reposition the same valve after re-collapsing (off-label maneuver), possibly completed by SCAP, (ii) use a different size of Perceval-S prosthesis, or (iii) switch to a sutured bioprosthesis.

End-Points

Primary end-points were implantation success and 30-day mortality ${ }^{16}$. 
Secondary end-points were postoperative stroke, AV-block requiring a permanent pacemaker and paravalvular leak, according to recommendation from Akins et al. ${ }^{16}$.

Perceval-S valve hemodynamics (effective orifice area, mean and peak gradients) were evaluated via transthoracic echocardiography (TTE), around post-operative Day 5 (MV, blinded for valve size, reviewed all TTE images) and patient-prosthesis mismatch was calculated according to Pibarot et al. ${ }^{17}$. We excluded hemodynamics of the patients in whom Perceval-S implantation finally failed.

\section{Statistical Analysis}

Statistical analysis was performed using SPSS BASE 17.0 statistical software (SPSS Inc. Chicago, IL, USA). Categorical variables were expressed as percentages and compared using the Chi-square or Fisher's exact test, as appropriate (n ? 5 per group). Continuous variables were expressed as mean +-1 standard deviation and were compared using the Student's t test. A two-tailed p-value less than 0.05 was taken to indicate statistical significance.

Results

Patients and Operative Data

During the observational period of the study, 115 consecutive patients underwent Perceval-S valve implantation in our institution and 103 were included in the present study. The 12 excluded patients (10\%) were emergency situations $(\mathrm{n}=5$, including four patients with endocarditis) and combined surgery other than AVR plus CABG $(\mathrm{n}=7$, including three AVR plus mitral, two AVR plus tricuspid and three AVR plus mitral and tricuspid procedures). Preoperative data are reported in Table 1. Fifty-seven patients (55\%) underwent isolated AVR, of which $17(30 \%)$ were performed via ministernotomy. The other 46 patients (45\%) underwent combined surgery with CABG (mean anastomoses 1.9). Mean CPB times were 59.3 +$45.4 \mathrm{~min}$ and 89.2 +- $43.5 \mathrm{~min}$, and aortic cross-clamp times were $39.4+-24.2 \mathrm{~min}$ and $69.0+-35.6 \mathrm{~min}$ in isolated AVR and combined procedures, respectively.

\section{SCAP Technique}

In total, 34 patients (33\%) required SCAP (Group SCAP); the other 69 patients $(67 \%)$ benefited from a classical implantation technique (Group No-SCAP). In Group SCAP, only one SCAP was performed in 23 patients $(68 \%)$ and a double SCAP in 11 patients (32\%). Perceval-S sizes and SCAP techniques are reported in Table 2 .

Moreover, the rate of SCAP's use differed according to the anatomy of the aortic valve, as SCAP was necessary in 24 patients (28\%) with a tricuspid aortic valve, versus in 10 patients (63\%) among those who had BAV (figure 2).

For patients who underwent in isolated AVR procedure, mean CPB times were $57.0+-38.3$ min and 64.6 +- $59.9 \mathrm{~min}$, and aortic cross-clamp times were $39.7+-26.4 \mathrm{~min}$ and $38.7+-18.7 \mathrm{~min}$ in groups No-SCAP and SCAP, respectively $(\mathrm{p}=0.57$ and $\mathrm{p}=0.90)$. This shows that SCAP did not significantly increase valve implantation or overall procedure times.

\section{Primary Implantation Failure}

Primary implantation failed in 14 patients (14\%) but finally 100 patients (97\%) benefited of a Percaval-S implantation. This very high rate of primary implantation failure (compared to current literature ${ }^{18-20}$ ) is explained because we considered any situations where a second valve deployment was required even if the same Perceval-S was reemployed. Fail situations are described in Figure 3.

In three patients $(21 \%)$, the valve exhibited an obvious instability after its deployment and was redeployed in good position before closing the aorta, with an additional SCAP in one patient. In the 11 other patients (79\%), a paravalvular leak was identified after weaning CPB, requiring a second clamping to attempt to settle this issue. In seven patients $(50 \%)$, the same Perceval-S was redeployed in good position, with an additional SCAP in three patients. In one case $(7 \%)$, the valve size needed to be increased from M to $\mathrm{L}$. 
In the three remaining cases (21\%), the Perceval-S valve was replaced by a sutured bioprosthesis (Trifecta St Jude Medical, MI, USA); in two of these, a total aortic root replacement had to be performed using a Freestyle aortic root bioprosthesis (Medtronic, Dublin, Ireland). Overall, SCAP enabled the implantation of Perceval-S in four patients (29\%) in whom the first implantation had failed.

\section{Clinical Outcomes and Post-Operative Hemodynamics}

The 30-day mortality rate was $2 \%(\mathrm{n}=2)$ for a EuroScore II predicted mortality of $2.95 \%$ (range 0.5 $16.5 \%$ ). Both of deaths were in Group No-SCAP (2.9\% of 69 patients) but only one was directly related to the procedure; the other one was related to the discovery of a multimetastatic lung cancer leading to an irreversible respiratory insufficiency and multi-organ failure.

There was no significant difference between the two groups regarding the different secondary end-points (table 3$)$. Only one patient ( $0.9 \%$ of overall) in Group SCAP (2.9\% of 34 patients) experienced a stroke with a persistent neurological deficit $(\mathrm{p}=0.40)$. Ten patients $(10 \%)$ presented a new onset of AV-block that required permanent pacemaker implantation, seven $(10 \%)$ and three $(9 \%)$ in Goup No-SCAP and Group SCAP, respectively $(\mathrm{p}=1.00)$. Only one $(0.9 \%)$ paravalvular leak more than trivial was found at the discharge TTE, in the Group No-SCAP (1.4\% of 69 patients) and it did not require reoperation.

Post-operative hemodynamics of the Perceval-S are shown in table 4 and in Figure 4. There was no significant difference between the two groups, but we noticed a trend towards lower peak and mean gradients in Group SCAP, which is consistent with a larger overall size of implanted valves. The rate of patient prosthesis mismatch was the same for the two groups.

\section{Conclusions}

For a decade, sutureless and rapid deployment aortic valves have provided a compromise between standard AVR and TAVI by favoring minimal surgical approach, reducing time procedure and limiting paravalvular leak. The sutureless Perceval-S aortic valve was designed to be surgeon-friendly and to make its implantation safe and reproducible. Our results corroborate its safety as early mortality was low and rate of implantation success, stroke, pacemaker requirement and post-operative hemodynamics were similar to those reported in the current literature ${ }^{19,21}$.

However, the sutureless feature of the Perceval-S is based on the specific design of an expandable nitinol stent which needs two anchoring sites: i) the first one, at the annulus level (inflow ring), ii) and the second one at the STJ level (outflow ring). These characteristics imply some procedural caution to avoid implantation pitfalls.

In order to prevent valve migration, the ratio between the aortic annulus and STJ diameters must be $<$ 1.3 , and the size of the aortic annulus must be $<27 \mathrm{~mm}$ (according that the Perceval-S sizes are : $\mathrm{S}=$ $19-21 \mathrm{~mm}, \mathrm{M}=21-23 \mathrm{~mm}, \mathrm{~L}=23-25 \mathrm{~mm}, \mathrm{XL}=25-27 \mathrm{~mm}$ ). Moreover, in order to minimize paravalvular leak occurrence, the aortic annulus must be decalcified enough (but avoiding annulus lesion) to be flexible enough to be conformed to the valve by the radial force of the Perceval-S stent. It is also important that annulus has a circular shape and that the heights of the sub-commissural triangles are neither too high nor too unequal. Furthermore, the height of the nitinol struts obligates to slightly modify the surgical technique by performing a horizontal and higher aortotomy than with sutured prosthetic valves, which could reduce the accessibility and the exposition of the diseased aortic valve.

Pre-operative imaging (cardiac-CT or echocardiography) is helpful to assess the shape and size of the annulus as well as to choose the best surgical approach ${ }^{22}$. That allows to estimate the likelihood of Perceval-S implantation success, but despite of this, some anatomic features of the aortic annulus (shape, flexibility, sub-commissural triangle height, calcification, etc...) are unpredictable before surgery and could hamper Perceval-S implantation. In such situations, the surgeon should typically substitute a sutured valve, which is more time consuming and may be challenging, especially through a minimally invasive approach ${ }^{1-4}$.

In order to address unexpected anatomical features of the annulus, some techniques of annuloplasty have 
been described sporadically to decrease the risk of implantation failure. In a short series, Ferrari et al. described how they sneaked a purse string suture of 3-0 polypropylene all around the aortic annulus before implanting a rapid-deployment aortic valve (Intuity, Edwards Lifesciences, Irvine, CAL, USA). Before closing the aorta, the suture was snared to tighten the implanted valve and minimize both paravalvular leak and valve migration ${ }^{23}$. In BAV, Glauber et al. suggested to reduce the height of the three sub-commissural triangles by a mattress suture before implanting a Perceval-S valve, if the annulus was overly scalloped ${ }^{15}$. In his series of 13 patients with BAV, Durdu et al. described a similar SCAP technique to ours, used in order to decrease the height of the sub-commissural triangles and to circularize the annulus ${ }^{14}$.

The present study highlights three situations in which SCAP was particularly helpful: (i) in case of aortic annuli that were slightly too large or too flexible (ii) in case of elliptic or overly scalloped aortic annuli (frequent in BAV), (iii) and as a rescue trick in case of implantation failure. We used 34 SCAPs successfully spread over these three situations, which permitted to widen the field of use of the Perceval-S. For example, among the 25 XL-sized valves implanted (including nine BAV), 14 SCAPs were performed, otherwise we would not have succeeded to implant it. This may partially explain why we reported a remarkable proportion of XL-sized valves compared to the current literature ${ }^{21,24}$. SCAP also afforded an issue to address implantation failure in almost one third of patients involved. We did not notice significant reduction of the effective orifice area surface, nor an increase of severe mismatch rates in the Group SCAP. Thus, we can infer that SCAP did not significantly reduce the annulus size but rather that it improved its congruence with the valve.

Our study presents several limitations. First, this is a monocentric retrospective study not in intention to treat, as the surgeon decided to implant a Perceval-S according to his feeling. Moreover, we did not randomize patients with a group control, in which we would use a sutured valve instead of SCAP if needed. Finally, our follow up was too short to evaluate the long-term stability of SCAP, especially in BAVs, which are known to dilate by time.

This study shows that SCAP is a safe and useful technique that could improve the success rate of implantation of the Perceval-S sutureless aortic valve essentially by circularizing the aortic annulus and reducing the height of sub-commissural triangles. It might be particularly useful in presence of a BAV or in case of a first implantation failure, as rescue trick. Larger studies and longer follow-up periods are necessary.

\section{Author contributions:}

AR and MK designed the study and operated patients. AR wrote the paper. AR, MV, MZG, PM, and VR collected data. MK performed statistical analysis. All authors critically revised and accepted the final version.

\section{References}

1. Meco M, Montisci A, Miceli A, et al. Sutureless Perceval Aortic Valve Versus Conventional Stented Bioprostheses: Meta-Analysis of Postoperative and Midterm Results in Isolated Aortic Valve Replacement. J Am Heart Assoc. 2018 Feb 16;7(4).

2. Mujtaba SS, Ledingham SM, Shah AR, Pillay T, Schueler S, Clark S. Aortic Valve Replacement with a Conventional Stented Bioprosthesis versus Sutureless Bioprosthesis: a Study of 763 Patients. Braz J Cardiovasc Surg. 2018 Apr;33(2):122-8.

3. Powell R, Pelletier MP, Chu MWA, Bouchard D, Melvin KN, Adams C. The Perceval Sutureless Aortic Valve: Review of Outcomes, Complications, and Future Direction. Innov Phila Pa. 2017 Jun;12(3):155-73.

4. Bilkhu R, Borger MA, Briffa NP, Jahangiri M. Sutureless aortic valve prostheses. Heart Br Card Soc. 2019 Mar;105(Suppl 2):s16-20.

5. Miceli A, Santarpino G, Pfeiffer S, et al. Minimally invasive aortic valve replacement with Perceval S sutureless valve: early outcomes and one-year survival from two European centers. J Thorac Cardiovasc Surg. 2014 Dec;148(6):2838-43. 
6. Baikoussis NG, Dedeilias P, Prappa E, Argiriou M. The perceval S aortic valve implantation in patients with porcelain aorta; is this ideal option? Ann Card Anaesth. 2017 Jan;20(Supplement):S70-2.

7. Gersak B, Fischlein T, Folliguet TA, et al. Sutureless, rapid deployment valves and stented bioprosthesis in aortic valve replacement: recommendations of an International Expert Consensus Panel. Eur J CardioThorac Surg Off J Eur Assoc Cardio-Thorac Surg. 2016 Mar;49(3):709-18.

8. Belluschi I, Moriggia S, Giacomini A, et al. Can Perceval sutureless valve reduce the rate of patientprosthesis mismatch?+. Eur J Cardio-Thorac Surg Off J Eur Assoc Cardio-Thorac Surg. 2017 Jun 1;51(6):1093-9.

9. Repossini A, Fischlein T, Solinas M, et al. Stentless sutureless and transcatheter valves: a comparison of the hemodynamic performance of different prostheses concept. Minerva Cardioangiol. 2018 Apr;66(2):18090 .

10. Santarpino G, Vogt F, Pfeiffer S, et al. Sutureless versus Transfemoral Transcatheter Aortic Valve Implant: A Propensity Score Matching Study. J Heart Valve Dis. 2017;26(3):255-61.

11. Miceli A, Gilmanov D, Murzi M, et al. Minimally invasive aortic valve replacement with a sutureless valve through a right anterior mini-thoracotomy versus transcatheter aortic valve implantation in high-risk patients. Eur J Cardio-Thorac Surg Off J Eur Assoc Cardio-Thorac Surg. 2016 Mar;49(3):960-5.

12. Lloyd D, Luc JGY, Indja BE, Leung V, Wang N, Phan K. Transcatheter, sutureless and conventional aortic-valve replacement: a network meta-analysis of 16,432 patients. J Thorac Dis. 2019 Jan;11(1):188-99.

13. PERCEVAL SUTURELESS AORTIC HEART VALVE Instructions for Use [Internet]. [cited 2019 Nov 14]. Available from: https://www.accessdata.fda.gov/cdrh_docs/pdf15/P150011d.pdf

14. Durdu MS, Gumus F, Ozcinar E, et al. Sutureless Valve Replacement Through a Right Anterior Minithoracotomy in Elderly Patients With Stenotic Bicuspid Aortic Valve. Semin Thorac Cardiovasc Surg. 2019 Autumn;31(3):458-64.

15. Glauber M, Ferrarini M, Lio A, Miceli A. Dealing with a stenotic bicuspid aortic valve: Is this still an off-label procedure for a sutureless valve? J Thorac Cardiovasc Surg. 2015 Oct;150(4):858-9.

16. Akins CW, Miller DC, Turina MI, et al. Guidelines for reporting mortality and morbidity after cardiac valve interventions. Ann Thorac Surg. 2008 Apr;85(4):1490-5.

17. Pibarot P, Dumesnil JG. Prosthesis-patient mismatch: definition, clinical impact, and prevention. Heart Br Card Soc. 2006 Aug;92(8):1022-9.

18. Laborde F, Fischlein T, Hakim-Meibodi K, et al. Clinical and haemodynamic outcomes in 658 patients receiving the Perceval sutureless aortic valve: early results from a prospective European multicentre study (the Cavalier Trial)+. Eur J Cardio-Thorac Surg Off J Eur Assoc Cardio-Thorac Surg. 2016 Mar;49(3):97886.

19. Shrestha M, Fischlein T, Meuris B, et al. European multicentre experience with the sutureless Perceval valve: clinical and haemodynamic outcomes up to 5 years in over 700 patients. Eur J Cardio-Thorac Surg Off J Eur Assoc Cardio-Thorac Surg. 2016 Jan;49(1):234-41.

20. Santarpino G, Berretta P, Fischlein T, et al. Operative outcome of patients at low, intermediate, high and "very high" surgical risk undergoing isolated aortic valve replacement with sutureless and rapid deployment prostheses: results of the SURD-IR registry. Eur J Cardio-Thorac Surg Off J Eur Assoc Cardio-Thorac Surg. 2019 Jul 1;56(1):38-43.

21. Sian K, Li S, Selvakumar D, Mejia R. Early results of the Sorin(r) Perceval S sutureless valve: systematic review and meta-analysis. J Thorac Dis. 2017 Mar;9(3):711-24. 
22. Margaryan R, Kallushi E, Gilmanov D, et al. Sutureless Aortic Valve Prosthesis Sizing: Estimation and Prediction Using Multidetector-Row Computed Tomography. Innov Phila Pa. 2015 Aug;10(4):230-235; discussion 235.

23. Ferrari E, Siniscalchi G, Tozzi P, von Segesser L. Aortic Annulus Stabilization Technique for Rapid Deployment Aortic Valve Replacement. Innov Phila Pa. 2015 Oct;10(5):360-2.

24. Concistre G, Chiaramonti F, Bianchi G, et al. Aortic Valve Replacement With Perceval Bioprosthesis: Single-Center Experience With 617 Implants. Ann Thorac Surg. 2018 Jan;105(1):40-6.

Tables:

Table 1. Patient preoperative demographics

$\mathrm{N}=103$

$\mathrm{N}=69$

$\mathrm{N}=34$

Gender (M/F)

Age

BMI

BSA

NYHA class

I-II

III-IV

Hypertension

Diabetes

Smoker

COPD

GFR $<30 \mathrm{ml} / \mathrm{min}$.

Logistic EuroSCORE II (\%)

$<4$

4-8

$>8$

Redo AVR

$\operatorname{LVEF}(\%)$

$>50$

$30-50$

$<30$

Peak gradient $(\mathrm{mmHg})$

Mean gradient $(\mathrm{mmHg})$

EOA (cm2)

Biscupid aortic valve

AVR, aortic valve replacement; BMI, body mass index; BSA, body surface area; COPD, chronic obstructive pulmonary dise

Table 2. Perceval S valve size and SCAP technique

Table 2.

$\mathrm{N}=103$

All

$\mathrm{N}=69$

Group $\Gamma$

Group A 
Table 2. Perceval S valve size and SCAP technique

$\mathrm{N}=34$

Perceval Size

Small

Medium

Large

$\mathrm{X}$-Large

SCAP technique

$\mathrm{NC} / \mathrm{LC}$

$\mathrm{LC} / \mathrm{RC}$

$\mathrm{NC} / \mathrm{LC}+\mathrm{NC} / \mathrm{RC}$

Native aortic valve

Tricuspid

Bicsupid

AV, aortic valve; LC, left coronary; NC, non coronary; RC, right coronary; SCAP, sub comissural annuloplasty.
Table 2.

$p$ value

$5(5 \%)$

$19(18 \%)$

$31(30 \%)$

$48(47 \%)$

$21(21 \%)$

$2(2 \%)$

$11(11 \%)$

$87(84 \%)$

$16(16 \%)$

$\mathrm{AV}$, aorti
Table 3. Postoperative clinical outcomes

$\mathrm{N}=103$

$\mathrm{N}=69$

$\mathrm{N}=34$

30-days mortality

Post-op hospital stay (days)

New AF

AV-block with PM

Stroke

LVEF (\%)

AF, atrial fibrillation; AV-block, atrio-ventricular block; LVEF, left ventricular ejection fraction.
Table 3. Postoperative

\section{All}

Group N

Group AP

$p$ value

$2(2 \%)$

$13.2 \pm 5.2$

$20(19 \%)$

$10(10 \%)$

$1(0.9 \%)$

$59.6 \pm 10.4$

$\mathrm{AF}$, atrial fibrillation; A
Table 4. Postoperative Perceval S valve hemodynamics

$\mathrm{N}=100$

$\mathrm{N}=67$

$\mathrm{N}=33$

Paravalvular leak $>$ trivial

Peak Gradient ( $\mathrm{mmHg}$ )

Mean Gradient (mmHg)

EOA $(\mathrm{cm} 2)$

PPM

None $>0.85$

Moderate 0.65-0.85

Severe $<0.65$

Missing

EOA, effective orifice area; PPM, patient-prosthesis mismatch.
Table 4. Postoperative Perceval S valve hemodynamics

All

Group N

Group AP

$p$ value

1 (1\%)

$21.7 \pm 7.5$

$11.8 \pm 3.9$

$1.73 \pm 0.52$

$0.93 \pm 0.28$

$46(54 \%)$

$29(34 \%)$

$11(13 \%)$

$14(14 \%)$

EOA, effective orifice area; PPM, patient-prosthesis mism 


\section{Figure legends}

Figure 1 . Aortic annulus remodeling by SCAP with a mattress suture 2-0 (green line) A . Reduction of the height of the sub-commissural triangle $\mathbf{B}$. circularization of an elliptic annulus. SCAP, sub-commissural annuloplasty.

Figure 2. Proportion of the number of required SCAP according to the valve type. SCAP, sub-commissural annuloplasty.

Figure 3. Patient flow chart. Among the 14 primary implantation failures, SCAP allowed for redeployment of the same Perceval-S valve in 4 patients (bold boxes). Same, re-deployment of the same valve; SCAP, sub-commissural annuloplasty.

Figure 4. Perceval-S valve hemodynamics by transthoracic echocardiography before discharge (postoperative day-5). EOA, effective orifice area; SCAP, sub-comissural annuloplasty; SCAP, no SCAP.
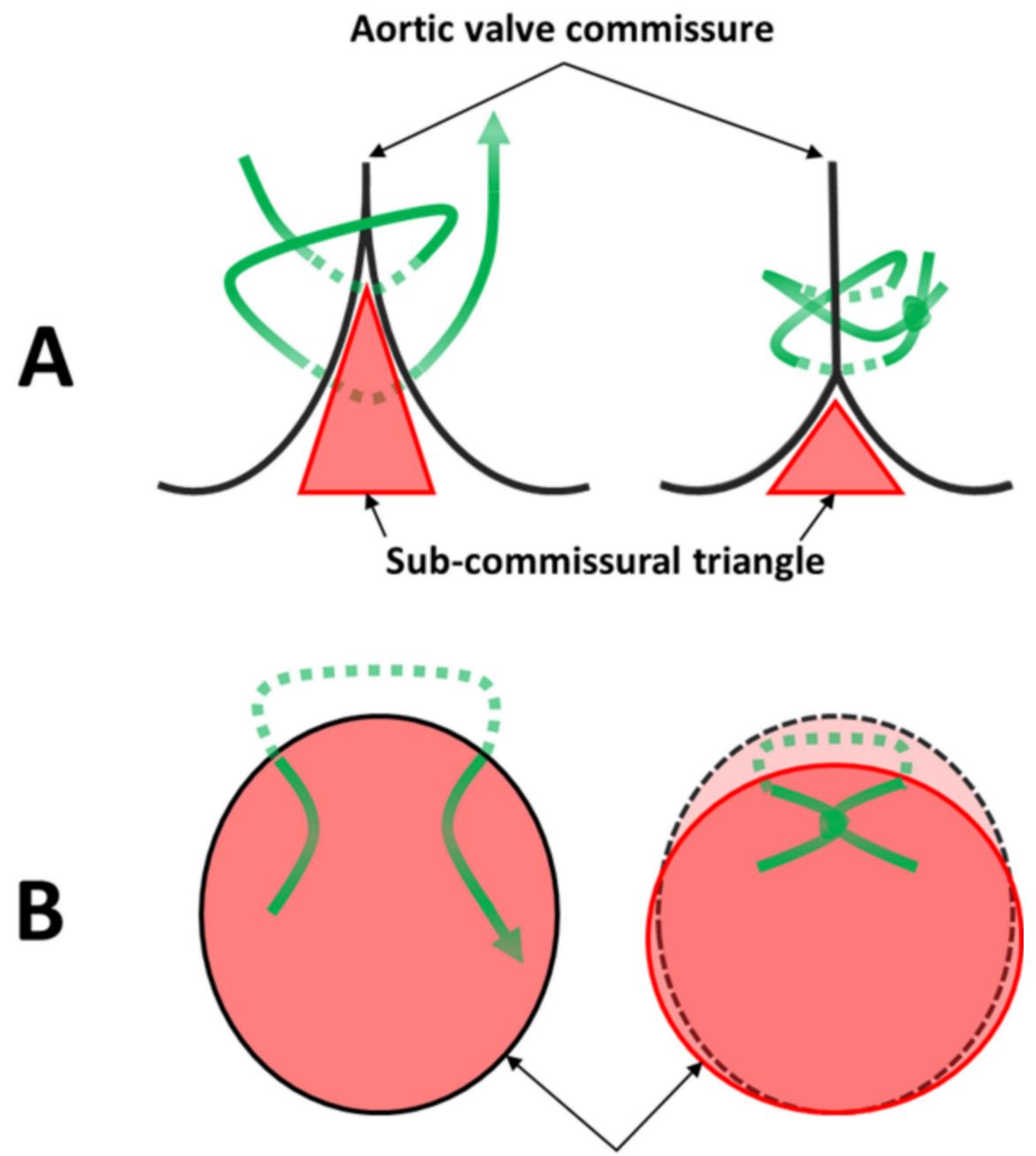

Aortic valve annulus 
Tricuspid

Bicuspid
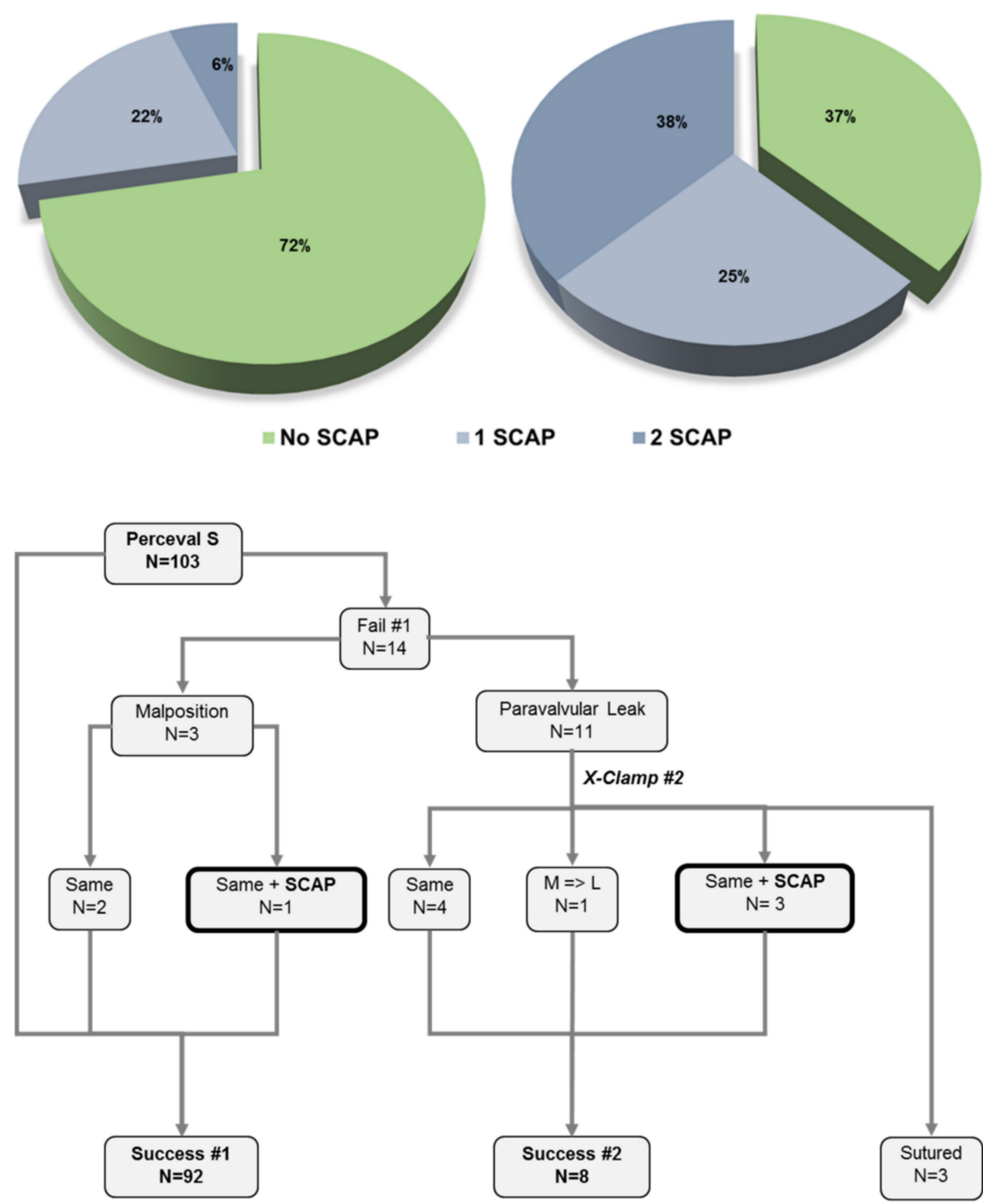
Mean Gradient (mmHg)
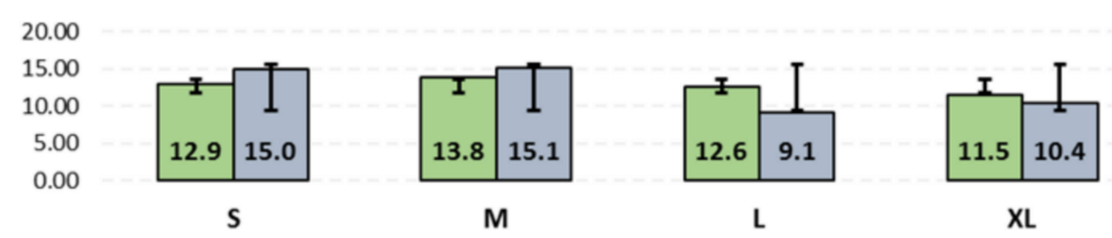

Peak Gradient (mmHg)


\section{$\operatorname{EOA}(\mathrm{cm} 2)$}

2.50
2.00
1.50
1.00
0.50
0.00
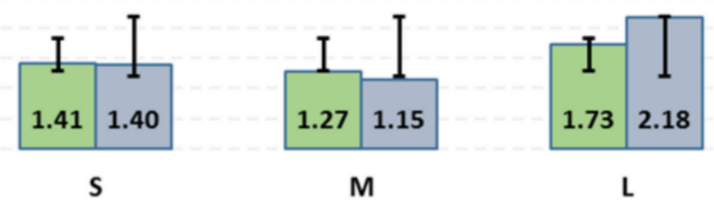

L

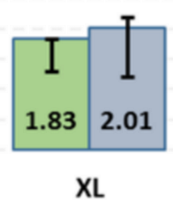

$\square$ No-SCAP $\square$ SCAP 